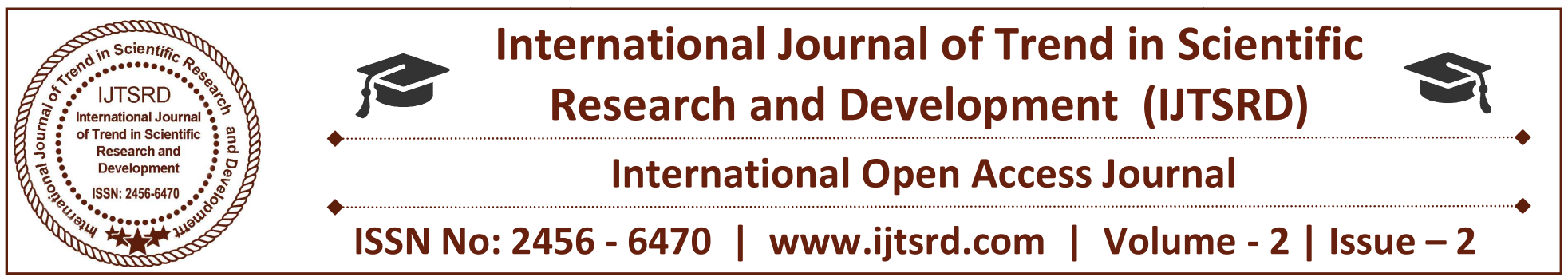

\title{
Autonomous Trajectory Tracking and Contouring Control of Three Dimensional CNC
}

\author{
Henna A \\ Student of Applied Electronics and Communication System Engineering, \\ Cochin College of Engineering and Technology, Valanchery, Malappuram, Kerala
}

\begin{abstract}
In this study we analyzed a three- axes computer numeric control (CNC) machine. Here improve the trajectory tracking and contouring performance of both linear and circular trajectories of a CNC. This paper introduced an autonomous method for better trajectory tracking and contouring by different position sensors are placed on three-axes of CNC machines. So reduce trajectory error and contouring error of CNC machines. This method is best solution for improve the trajectory tracking ability and precision of a $\mathrm{CNC}$ machine for both linear and circular trajectories.
\end{abstract}

Keywords: Computer numerical control (CNC), Autonomous, Trajectory tracking, Contour, Contouring error

\section{INTRODUCTION}

Computer numerical control (CNC) machines tools are now widely used in manufacturing industry. With an increasing demand on the accuracy of the CNC machines, now we are research for various method to improve the accuracy of CNC machines. Contouring accuracy in terms of contour error is a big concern for designers and contouring type of CNC machines. Contouring error is defined as the deviation of actual contour from that desired contour.

In CNC machines, a part is manufactured by a part program which is tool type and feederate. The programme can be written by computer-aided manufacturing (CAM) or manually written.

This paper is improved the trajectory tracking ability of a CNC machines for both circular and linear trajectories by using position sensors. There are different sensors are used according to their applications. There are different controller approaches are also used for better trajectory tracking, that is proportional-integral -derivative (PID), observer based PID and feedforward computed torque (FFCT). Although computing time increases, the autonomous method is found as the best solution for the considered case to improve the trajectory tracking ability of the manipulator for both liner and circular trajectories.

In manufacturing system, the precision motion control is a very importance, especially in CNC machines. For precision motion control at $\mathrm{CNC}$ machines in all axes must be controlled simultaneously. Axial tracking errors may cause simultaneously coordination of axes. This coordination problem between axes can cause for contour errors, that is it is the shortest distance between actual path and desired contour. The best way to reduce tracking error is to improve tracking accuracy of each axis individually. This is done by taking into account their dynamic interaction tracking control approaches to improve not only tracking accuracy but also contouring accuracy.

Necessity of high precision CNC machine tool system has been motivating advanced model-based control approaches to machining process with high tolerance, better surface finishing and high speed. The surface finishing is directly related to the deviation of the 
cutting tool from the tool path trajectory. This deviation is contouring error, it measure the cutting process quality. In the tracking control, the objective is to minimize the tracking error in each axis separately, which cause for small contouring errors. Small contouring errors can be achieved even when it is impossible to attain small tracking errors due to saturation of servo motor. Contouring control techniques have been developed to take advantage of this fact, by placing higher priority in minimizing contouring error than tracking errors.

\section{A. Contouring and tacking accuracy}

The contour accuracy is the deviation of the actual path from the desired path. At any point on the path, it is defined it is the orthogonal distance between actual path and desired path.

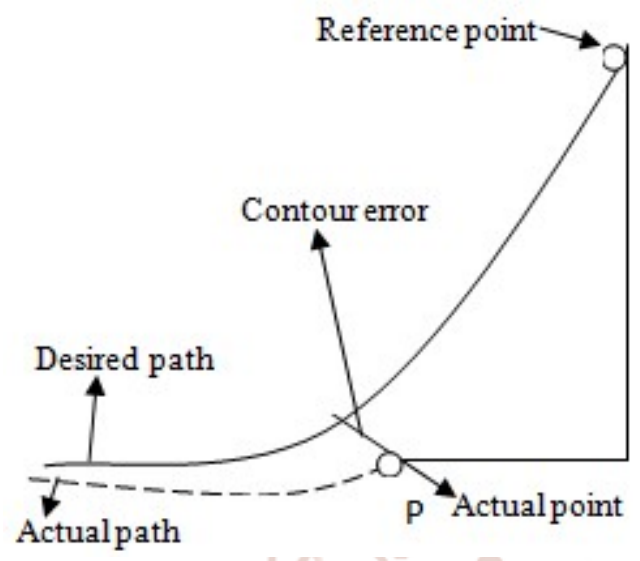

$R$

Fig. 1 Contour error in $\mathrm{CNC}$ machines
Force in the early 1950s, after that computer numeric control was developed. In $\mathrm{CNC}$ monitor the movement of machines based on CAM/CAD programmes. The machine can be milling, lathe, router, laser or waterjet cutter, robot etc.

The CNC controller work together with a series of motors and drive components to move and control the machine axes, executing the programmed motions. In $\mathrm{CNC}$ our desired model drawing using any type of CAD programme in computer and at three axes there was thee different stepper motor for each axis motion of this axis is based on the motion of the stepper motors the computer connect to the interface, it will help the signal from the computer to a form that the CNC machine can use. That mean our desired model drawing is decoded to G-code for CNC can read only G-code or ISO code. The signal from the interface control the movement of the cutting tool A CNC machine tool is always a slide table and spindle. The machine table is controlled the $\mathrm{X}$ and $\mathrm{Y}$ axis motion and spindle is controlled $\mathrm{Z}$ axis motion. The Fig.2 shows the 3 axes CNC machine.

IT $S \begin{aligned} & \text { and spindle is controlled } Z \text { axis motion. The Fig.2 } \\ & \text { shows the } 3 \text { axes CNC machine. }\end{aligned}$

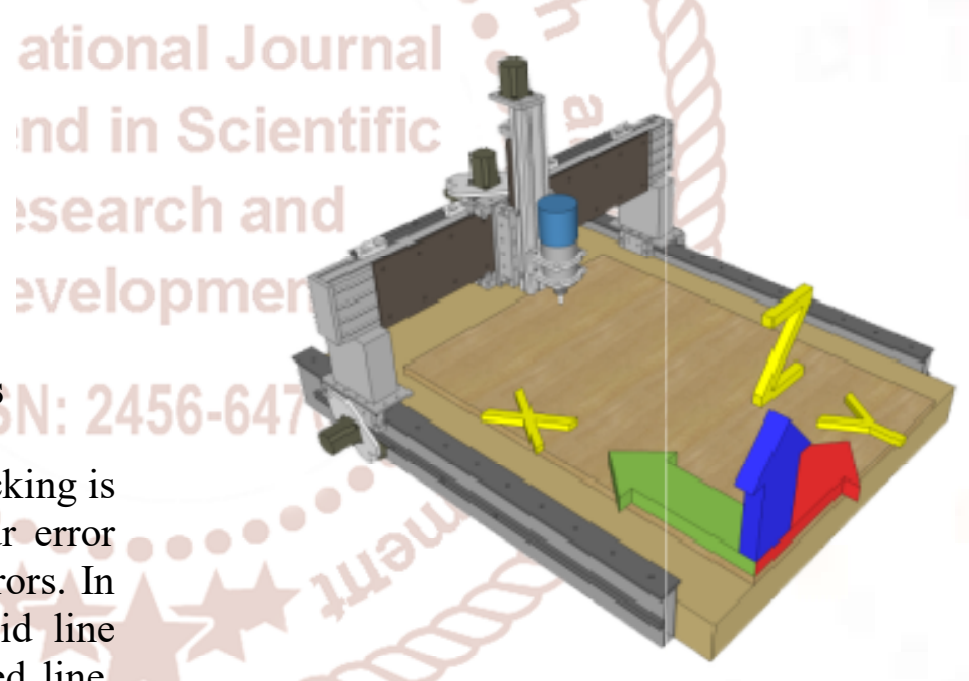

Fig. 2 Three axes CNC machine

\section{III.LITERATURE SURVEY}

In this section, some controllers used for feed drives to achieve better performance of $\mathrm{CNC}$ machines are presented and discussed. Controlling a machine tool by a prepared program which is numerical data specifying the desired motion of the machine's axes is called as numerical control (NC). $\mathrm{NC}$ is the result of a research sponsored by the US Air Force in the early 1950 s, when there was a demand for variable methods to manufacture complicated and accurate parts for aircrafts. 
The feedforward controllers are used to control the error. The basic idea iof feedforward controller is to close the servo loop through a feedback controller to take care of disturbances and parameter variations, and to cascade the closed-loop dynamics with a feedforward controller with a gain such that it cancels out most of its stable components.

While tracking control aims to improve the tracking accuracy of individual axes, the cross-coupled controller (CCC) is other method is devised to reduce the contour errors directly, rather than achieve this by reducing the individual axial tracking errors. In crosscoupled control is to particular real time a contour error model based on the feedback information from all axes and then to feed back correction signals to the individual axes.

In order to compare the effectiveness of the different control approaches on the improvement of trajectory tracking ability and contouring performance of the three-axes CNC, optimally tuned PID, observer combined PID and FFCT control approaches have been applied. To improve the performance of the PID controller, a Luenberger observer has been designed, and design steps have been given in detail. Finally, the FFCT control method has been designed to reduce the effects of nonlinear coupling inertia, centripetal, and gravitational forces and also reduce trajectory tracking error

\section{IV.PROPOSED SYSTEM}

In this system an autonomous CNC machine. For autonomous control we use position sensors, that are placed on $\mathrm{X}, \mathrm{Y}$ and $\mathrm{Z}$ axis. Have an continues monitoring the output voltage of sensor and any error is occurred when the output voltage of sensor is changed then sensors output is gives to controller the an error correction comment is passed to stepper motor of $\mathrm{CNC}$

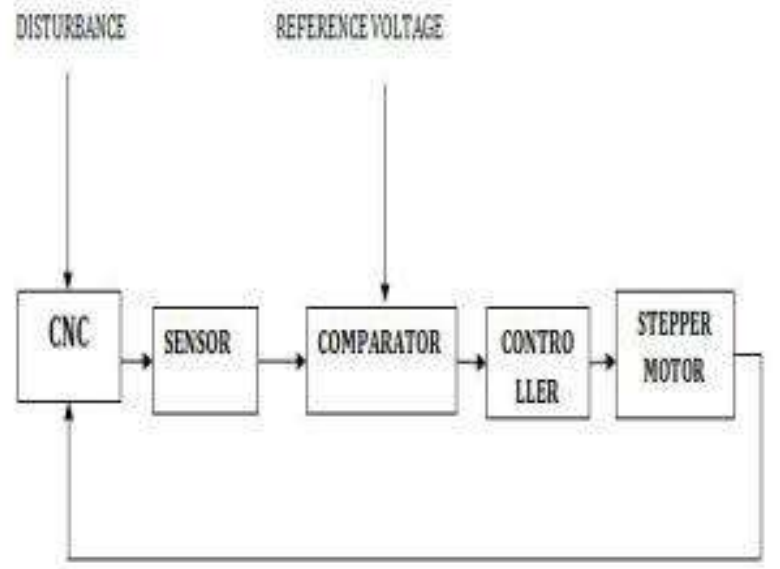

Fig. 3 Block diagram of proposed system

In fig. 3 The disturbance experienced on any axis of $\mathrm{CNC}$ the corresponding axis sensor change it output voltage or compare the sensor output voltage with reference voltage this error is gives to controller then command send to stepper motor when stepper motor control that motion.

\section{HARDWARE PART}

In hardware system, an IR sensor (FC-51) used as position sensor placed on three axes. The sensor is connected to our controller.

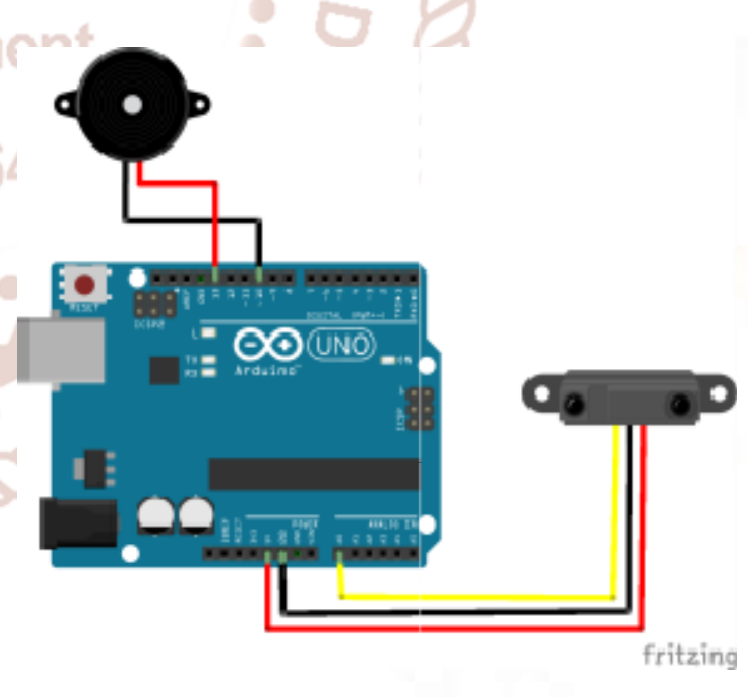

Fig. 4 Sensor and motor on controller

From Fig.4 we can understand sensor and motor are connected to controller so has any change occurring in sensor output when corresponding control is gives to stepper motor.

\section{RESULT}


In this study we analyzed the contouring and trajectory tracking of a CNC machine. In this paper implemented the hardware of proposed system. By using this sensor feedback method we can reduce the contour and trajectory tracking errors. So a well performance $\mathrm{CNC}$ machine can be designed.

\section{CONCLUSION}

In this study, a three-axes CNC machine is also known as portal type CNC machine has been analyzed, and its trajectory tracking control for linear and circular trajectories has been examined. The proposed system improve trajectory tracking control and contour performance.

An autonomous method is help to improve the precision of CNC machine and reduce the wastage of work piece also help to time saving of a manipulator.

\section{ACKNOWLEDGMENT}

The original idea for this work is came from a paper is 'Trajectory tracking control and contour performance of three dimensional CNC' by M. Fatih
Corapsiz and Koksal Erenturk, Member, IEEE . so our special thank to this paper's authors

\section{REFERENCE}

1. C. L. Chen, M. J. Jang, and K. C. Lin, Modeling and high-precision control of a ball-screw-driven stage, Precesion Engineering 28 (2004), no. 4, 483-495.

2. Syh-Shiuh Yeh and Pau-Lo Hsu, Analysis and design of the integrated controller for precise motion systems, IEEE Transactions on Control Systems Technology 7 (1999), no. 6, 706-717.

3. Xue-Cheng Xi, Aun-Neow Poo, and Siaw-Kiang Chou, Factors affecting contour errors in CNC systems, Proceedings of 2005 9th International conference on mechatronics technology (Kuala Lumpur, Maylasia), 2005, pp. ICMT 117.

4. Syh-Shiuh Yeh and Pau-Lo Hsu, Analysis and design of the integrated controller for precise motion systems, IEEE Transactions on Control Systems Technology 7 (1999), no. 6, 706-717. 